\title{
Engineering the Energy Flow in Nanoparticles for Plasmonically Enhanced Catalysis
}

\author{
Canhui Wang ${ }^{1,2}$, Wei-Chang D. Yang ${ }^{1,2}$, Devika Sil ${ }^{1,2}$, Amit Agrawal ${ }^{1,2}$, and Renu Sharma ${ }^{1}$ \\ 1. Center for Nanoscale Science and Technology, National Institute of Standards and Technology, \\ Gaithersburg, USA. \\ 2. Maryland NanoCenter, University of Maryland, College Park, USA.
}

Excitation of localized surface plasmon (LSP) modes in metal nanoparticles has been shown to reduce the energy barrier for photochemical reactions, even allowing certain reactions to occur at room temperature. Recently, hybrid plasmonic-catalytic systems have been proposed as an efficient route towards channeling energy from a plasmonic nanoparticle to the catalytic metal [1-2]. However, the stochastic nature of catalyst sites on the nanoparticle surface does not allow efficient energy flow pathway from a localized plasmonic hotspot of reaction relevant energy. Moreover, a mechanism to modify the location of plasmonic hotspots to overlap with the catalytically active sites is still missing. Here we demonstrate spatially localized and selective transfer of energy into and out of a nanoparticle using a non-uniform thickness coating of dielectric layer. This approach allows modification of the local plasmonic response at nanometer scale based the location of the catalytic active sites, and can be used in nanoparticle engineering to achieve an optimal catalytic efficiency.

We use $\mathrm{Al} / \mathrm{Al}_{2} \mathrm{O}_{3}$ core-shell nanoparticle as a model system to investigate the effect of non-uniform coverage of dielectric material on modulating the energy flow pathways during the excitation of an LSP resonance, and the subsequent LSP induced $\mathrm{H}_{2}$ dissociation reaction. An ensemble of techniques is used to characterize various aspects of the LSP-promoted chemical reaction in an environmental transmission electron microscope equipped with a monochromated electron source. Electron energy-loss spectra (EELS) imaging, with different energy dispersions, is used to acquire both elemental and LSP maps from the same particle. Combined with non-negative matrix factorization (NMF) machine learning algorithm [3], we map the energy transfer pathways from the excitation source to the nanoparticle at nanometer spatial resolution and $0.08 \mathrm{eV}$ energy resolution (Fig. 1). Figure 2 shows characterization of a nanoparticle before and during $\mathrm{H}_{2}$ gas is introduced. By comparing the EELS spectra of the nanoparticle acquired in vacuum and in $\mathrm{H}_{2}$ environment, the energy and the location for the energy transfer during the LSPR promoted hydrogen dissociation reaction is measured (Fig. 2G). The location of the energy transfer on the nanoparticle surface in the $\mathrm{H}_{2}$ environment (Fig. 2F) overlaps with the location of the reaction-relevant energy absorption measured in vacuum (Fig. 2C). These results show that the dielectric $\mathrm{Al}_{2} \mathrm{O}_{3}$ layer thickness modifies the local absorption coefficient of the excitation energy, and therefore determines the location of dissociation on the nanoparticle. In addition, $\mathrm{H}_{2}$ adsorption map shows that the added $\mathrm{Al}_{2} \mathrm{O}_{3}$ dielectric layer acts as a gas adsorption and accumulation layer. This is likely due to the porous morphology of $\mathrm{Al}_{2} \mathrm{O}_{3}$ which allows the gas molecule to reach the $\mathrm{Al}_{2} / \mathrm{Al}_{2} \mathrm{O}_{3}$ interface and increases the local gas molecule concentration surrounding the plasmonic Al core. Our results demonstrate that a thickness controlled dielectric additive layer can be used to control the energy flow pathways in the nanometer scale, which will allow sub-particle control of LSP promoted reactions [4].

\section{References:}

[1] U. Aslam, S. Chavez, and S. Linic, Nature nanotechnology, 12(10) (2017), p. 1000. 
[2] D. Swearer, et al. Proceedings of the National Academy of Sciences 113.32 (2016), p. 8916.

[3] O. Nicoletti, et al. Nature $\mathbf{5 0 2 . 7 4 6 9}$ (2013), p. 80.

[4] The authors acknowledge funding from the Cooperative Research agreement between the University of Maryland and the National Institute of Standards and Technology Center for Nanoscale Science and Technology, Award 70NANB14H209, through the University of Maryland.
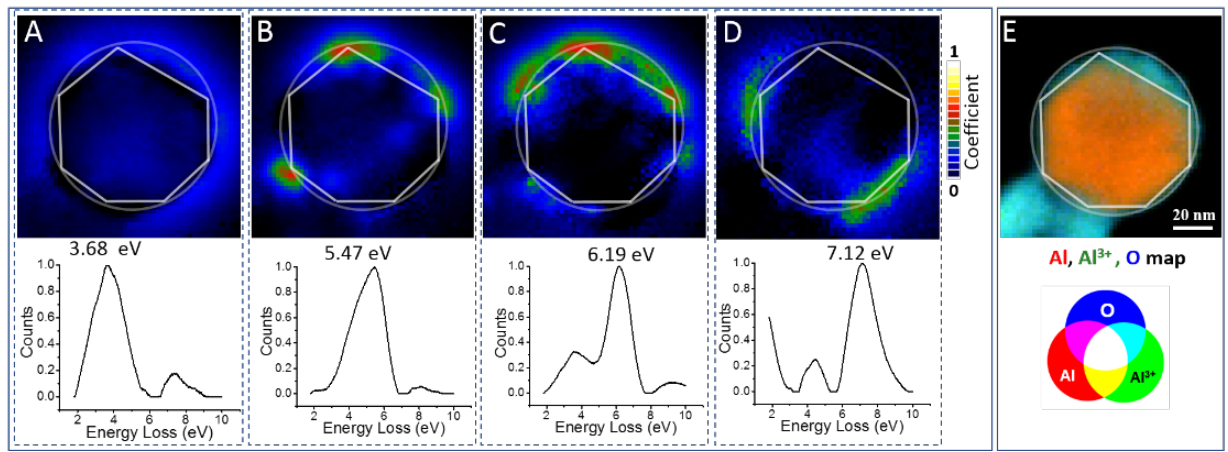

Figure 1. A-D, Principle components of the EELS as well as their loading coefficient acquired from NMF algorithm, showing the energy and spatial distribution of the energy transferred to the nanoparticle. E, Elemental map of an $\mathrm{Al}$ nanoparticle with a non-uniformly covered $\mathrm{Al}_{2} \mathrm{O}_{3}$ shell. Comparison of $\mathrm{C}, \mathrm{D}$ and $\mathrm{E}$ shows the effect of the local $\mathrm{Al}_{2} \mathrm{O}_{3}$ thickness on the distribution of the energy transfer. Higher local $\mathrm{Al}_{2} \mathrm{O}_{3}$ thickness can be associated with lower LSPR energy.
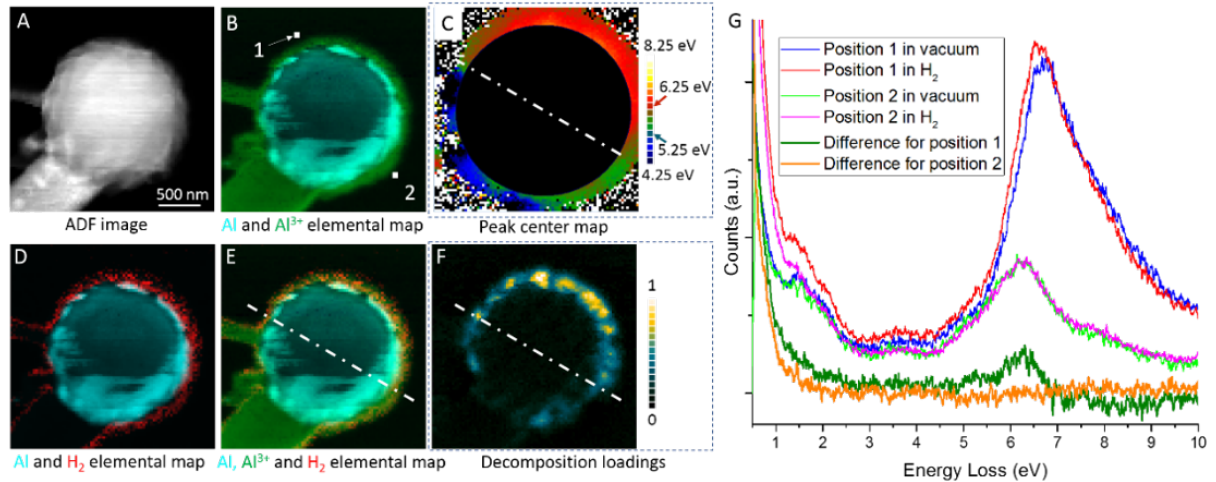

Figure 2. A, Annular dark field (ADF) image of an Al nanoparticle. B, D, E, Selective elemental maps of the nanoparticle in vacuum (B) and in $\mathrm{H}_{2}$ environment (D, E). The $\mathrm{H}_{2}$ signal from the background is subtracted from the entire map in $\mathrm{D}$ and $\mathrm{E}$. A residual $\mathrm{H}_{2}$ signal shows the accumulation of the $\mathrm{H}_{2}$ within the surface $\mathrm{Al}_{2} \mathrm{O}_{3}$ layer. $\mathbf{C}$, Peak center map of the most dominant LSP peak of the nanoparticle in vacuum. The increase of the peak energy at the upper right region can be attributed to the presence of the $\mathrm{Al}_{2} \mathrm{O}_{3}$ nanorod at the lower left corner. $\mathbf{G}$, Comparison of the EELS spectra acquired at position 1 and 2 shown in B. The difference of the spectra acquired at position 1 between vacuum and $\mathrm{H}_{2}$ environment is shown in green, which exhibits a peak with center at around $6.25 \mathrm{eV}$. This peak can be attributed to the direct electron transfer process during the $\mathrm{H}_{2}$ molecule dissociation. F, Normalized decomposition loadings of the principle component that contains the additional $6.25 \mathrm{eV}$ peak in $\mathrm{H}_{2}$ environment, acquired via $\mathrm{NMF}$ algorithm. 